\title{
Parallel Tracking and Mapping of a Fleet of Quad-Rotor
}

\author{
M. Bazin, I. Bouguir, D. Combe, V. Germain, and G. Lassade
}

\begin{abstract}
The problem of managing a fleet of quad-rotor drones in a completely unknown environment is analyzed in the present paper. This work is following the footsteps of other studies about how should be managed the movements of a swarm of elements that have to stay gathered throughout their activities. In this paper we aim to demonstrate the restrictions of a system where absolutely all the calculations and physical movements of our elements are done by one single external element. This is made possible thanks to a set of command rules which can guide the drones through various missions with defined goal. This strategy is based on a simultaneous usage of different data: obstacles positions, real-time positions of all drones and relative positions between the different drones. This work is based on Robot Operating System (R.O.S.) and use, as a base, several previous open-source projects on the matter. Simulation results show the limitations of the use of a centralized system to control a fleet of drones. Those weaknesses are highlighted thanks to the low-cost drones used here where the central unit had difficulties to handle drones workload.
\end{abstract}

Index Terms-Autonomous multi-vehicles system, Cooperative guidance, distributed control, obstacle avoidance, unmanned aerial vehicle.

\section{INTRODUCTION}

Flying drones is a relatively recent technology so that the research in this field is not very advanced. The success of unmanned vehicles, like drones, and the possibilities we can predict for them make those researches an important and interesting matter.

The aspects developed in present paper about drone technologies is the collaborative motion of drones in an open environment, with basic tools to evaluate the position and at least one on board camera on the drone [1], [2]. Present concern is to control a fleet of drones to guide them to a goal with avoidance of possible obstacles and without collision with other fleet members [3], [4]. Flying fleets of quad-rotor drones is not developed yet but it implies interesting elements for general multi-component systems. Indeed, flying with a fleet of drones can increase the success rate of a mission: one can allocate specific functionalities to each drone to give the fleet the needed tools for a particular mission for reduced drone price by using cheaper materials, whereas the global performance throughout the mission is preserved thanks to the optimized sharing of workload between the different fleet members.

Once a task has been assigned, there is a large technical freedom in designing the multi-drone system supposed to

Manuscript received January 26, 2016; revised May 1, 2016.

M. Bazin, I. Bouguir, D. Combe, V. Germain, and G. Lassade are with ECE Paris School of Engineering, Paris, France (e-mail: vgermain@ece.fr). perform it, from unique super-equipped one single drone to a distributed multi-drone fleet where each member is assigned functionality. In this last case, an important issue in fleet coordination is the adequate split between centralized vs. self-organized organization depending on embarked possibilities on-board each drone. Indeed, it is observed that using robust swarm intelligence approach reduces overall cost and permits miniaturization of individual robots. These two aspects are key-factors for drone democratization in the civilian market. Many studies have already been undertaken in multi-component system about swarm or collective behaviors emerging from interactions between components themselves and/or with the environment. It is chosen here to focus on guidance law which allows drones fly as a fleet, similar to a flying animal flock. Such study can be meaningfully developed on low cost drones with modest equipment compatible to usual constraints in an academic environment.

The research about guidance laws to control small aerial vehicles comes from two main methods: using three behaviors to manipulate drones movement [5] or using predictive guidance [6]. The first method use three coefficients, simulating three different drone behaviors: 'attraction', 'imitation', 'repulsion'.

\section{Algorithms: OBSTACLES, REAL-TIME LOCALISATION AND FORMATION}

Gathering data in real time is a key point in the project. Indeed, an important part is to manage a fleet of drones through different distances while avoiding all obstacles, for which it is clear that the need of a system fast and flexible enough to ensure drone safety at all times and for any given situation (Fig. 1).

Those data are vital for the project and are gathered through the usage of a mapping and localization algorithm called SLAM [7]-[14] (Simultaneous Localization And Mapping).

Present project aims at developing a program to manage a fleet of drones in an unknown environment. A requirement is that every drone has to be able to move autonomously. SLAM is based on the video feed from frontal cameras embarked on board the drone (as for example on Parrot AR Drone), this is aprerequisite for the system to work. At the end the output is a cloud of points which can be represented in a $3 \mathrm{D}$ map[15].

Each point represents a difference of depth, usually on the border of objects. SLAM can provide a trustworthy map of environment. This map can be updated in a real time and help to detect obstacles on the path of drones and simultaneously provide an estimation of respective positions of all fleet members. This feature can ensure the fleet cohesion at all times. 


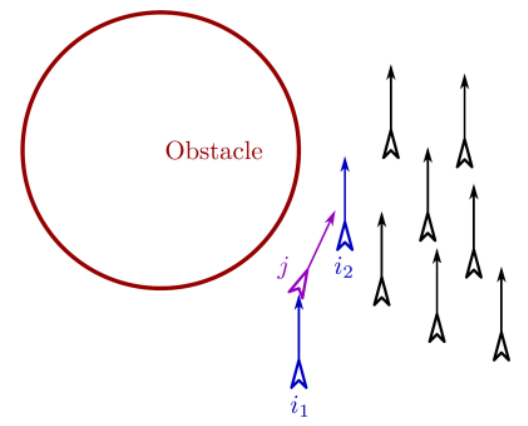

Fig. 1. Contribution of vehicle $j$ to the command of vehicles $i 1$ and $i 2$. Near obstacle, $\mathrm{j}$ deviates to the right, $\mathrm{i} 1$ is anticipating obstacle avoidance by mimicking j. Same behavior goes for $i 2$ to leave enough space for $\mathrm{j}$.

\section{Helpful Virtual Agent System, Obstacle MANAGEMENT, FLEET COHESION}

In an unknown environment, it is impossible to know most outside parameters like power or even wind direction. These environmental parameters create scenarios where the drones can collide. The probability that a collision between drones happens is proportional to fleet size.

To solve this important issue several options can be implemented to minimize the risks. One possible way is to use a powerful guidance law for the drones, such as the rule of "nearest neighbor" which reduces by a sensible amount the risk of collision in cases where two drones are going to the same point from different sides. This alone is not sufficient, and other algorithms are required, based on virtual agents influencing the whole fleet through different coefficients, and proportionally to the distance between the agents and the drones, see Fig. 2.

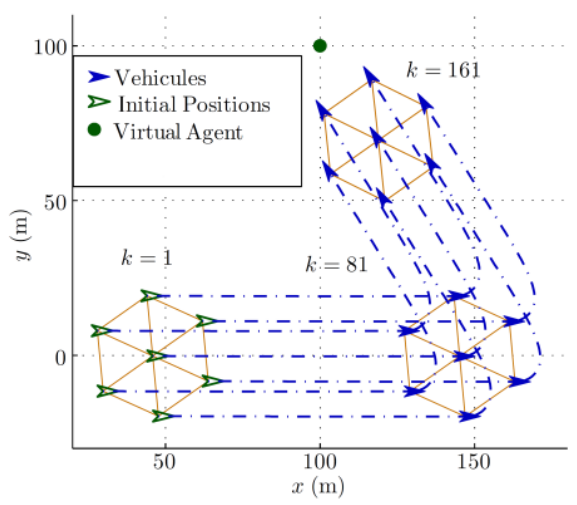

Fig. 2. Use of virtual agent to create a goal for the vehicles.

All fleet members are agents as well as virtual agents 'guiding' to a goal or just giving a general direction for the fleet to follow.

All agents are defined by their position, speed vector and direction. These parameters are calculated at each iteration from equations based on coefficients given by the distance between agents [5], [6].

Although the computer where these algorithms are processed is the only decision maker of the system, each virtual agent is autonomous and has its own data about its environment. This centralized intelligence approach allows avoid difficulties caused by using low-cost quad-rotor. Indeed, by making iterations on individual drone path and then computing the fleet progress really improves mission global trajectories and duration without expensive on-board materials.

\section{MATERIALS}

In present study Parrot AR Drone V1 and V2 have been used [16]. Both versions are equipped with two cameras (frontal and vertical ones), one altimeter, one gyrometer and one accelerometer. Ad hoc network has been first used to connect multiple drones to a computer, and after on router network to make it more stable. Both network structures are possible.

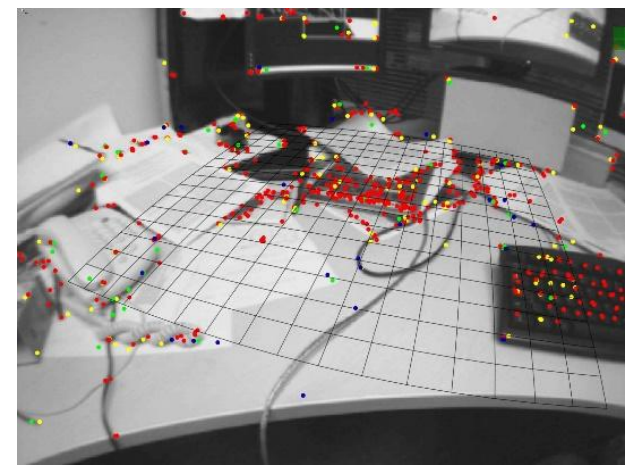

Fig. 3. Point Cloud generated by PTAM program. The points are transferred to another map for managing multiple virtual agents.

In term of programming, present work is based on $\mathrm{AR}$ Drone autonomy and TUM_ARDRONE software [9], [17], two "packages" of R.O.S.. First one is the first layer between Parrot AR-Drone and the programmer. It can send and receive basic packets from the drone, and is used to get measurements from the various sensors and to send the commands. Second one TUM_ARDRONE is a package using PTAM [18] (Parallel Tracking and Mapping), another package made for mapping with little robots/drones, see Fig. 3. TUM_ARDRONE is the implementation of PTAM on Parrot AR Drone (V1 or V2). This program comes with one interface but it cannot manage multiple drones and do not exploit full potential of PTAM[19]. The realized C++ program is the effective use of PTAM on multiple AR Drones, coupled with previously introduced Agents algorithms [5], [6].

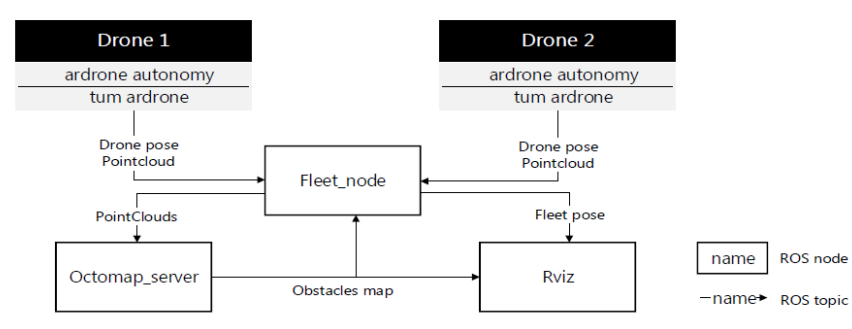

Fig. 4. System architecture and links between nodes with data exchange.

In order to obtain a cooperative PTAM wherein drones can share their map with each other, it has been chosen to use OCTOMAP library [20], a dynamic 3D occupancy grid mapping framework. OCTOMAP uses a probabilistic occupancy estimation approach which aims to reduce sensors measurements noise and improve the accuracy of generated 3D map. Furthermore, it is configured to avoid the storage of useless data like points which are below drone minimum altitude and/or out of mission range. 
In adapting previous programs equations and principles are not modified, but the commands directly send to the drones are simpler. For the moment and for safety purposes, only one speed is used and the drone can only turn on its yaw axis. Corresponding rotation is only to acquire the desired angle the drone must be facing according to the calculations performed by the algorithm.

\section{TESTS AND RESUltS}

\section{A. Goals and Conditions}

The purpose of performed tests was to experiment robustness of both centralized and self-organized fleet behavior to determine which one of the central unit or the drones is system more important part. To this aim, tests have been made in two different ways, first missions with PTAM tracking where each drone brings its contribution to central unit on its environment, secondly without PTAM where drones bring less information and where guidance algorithm (swarm behavior) takes a more important part in success probabilities.

\section{B. Material}

The working fleet contained AR Drones V1 and V2 disposed in a particular manner for each flight, see Fig. 5.

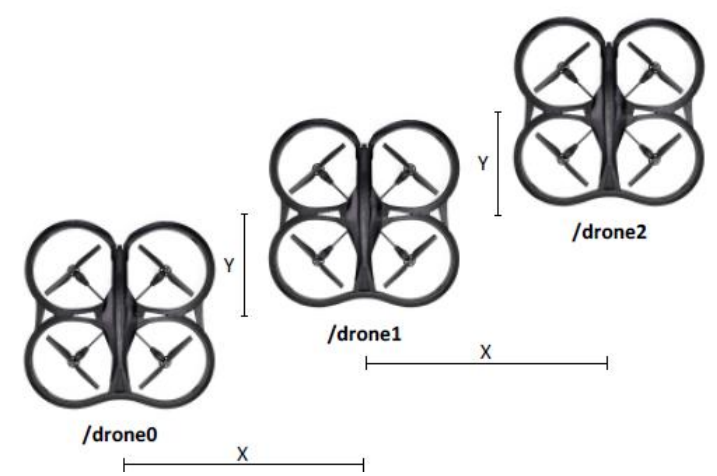

Fig. 5. Recommended drones initial positions.

Distances between the drones are calculated to insure material safety and a common behavior for the drones[21].

\section{Mission Description}

For testing purpose and for material safety, tests have been performed in indoor environment. The major goal of these tests was to evaluate the correctness of adaptation capacity of adaptation of used algorithm [5], [6], [22]. During the tests, different factors have been varied: initial drone position, drone target coordinates (between 3 to 7 meters) and layout of possible obstacles. Twenty two different missions have been worked out, thirteen with a PTAM and nine without it. Results are represented in Table I below.

\section{Results}

Due to wrong measurements. Indeed, the tool TUM_ARDRONE happens to create points (later transformed into obstacles) right in front of drone, causing major problem in the trajectory[23]. This can however be significantly reduced by adjusting OCTOMAP filtering parameters. For missions without PTAM, obstacles coordinates have been manually input, increasing trajectory accuracy and producing higher success rate. Inertial drone central station is highly subjected to noise and so estimated positions by these sensors alone are extremely imprecise. This fact leads to higher risk of collision between fleet members. Interestingly, chosen technologies clearly show the limits of totally centralized system. Video stream takes too much WIFI bandwidth and the saved resources at each drone level have to be managed by central unit, causing latency impacting probability of success. One way to improve system robustness is to limit the number of drones with PTAM and to share functions between drones by assigning to them specific roles of headers and followers, in other words, to distribute decision between fleet members.

TABLE I: TEST RESULTS IN INDOOR ENVIRONMENT WITH GOALS LOCATED IN INTERVAL 3-6 METERS FROM FLEET INITIAL POSITIONS STYLES

\begin{tabular}{|l|l|l|}
\hline & With PTAM & $\begin{array}{l}\text { Without } \\
\text { PTAM }\end{array}$ \\
\hline Mission Success Ratio & $61 \%$ & $77 \%$ \\
\hline Mission Success Ratio with Obstacle & $50 \%$ & $75 \%$ \\
\hline Without Obstacles & $80 \%$ & $80 \%$ \\
\hline $\begin{array}{l}\text { Error Range of Final Distances } \\
\text { (meter) }\end{array}$ & $0.2-0.8$ & $0.5-1.3$ \\
\hline
\end{tabular}

\section{CONCLUSION}

Though obtained with modest quality equipment, present results already show that the delays led by centralized calculations are increasing final position discrepancies between the flights, and at the same time, reduce significantly probability of mission success. As expectable centralized guidance directly adds delays between acquisition of information by the drones and their actual displacement based on this information. To a certain point the drones are able, without using a centralized system, to take into account their environment and adjust their position based on it. So present approach where all the calculations are centralized in one place is not the best way to handle the fleet control problem. Indeed, as experimentally shown, the drones are fully capable to flight autonomously to a certain extent, when managing to share calculation workload between the different fleet members. Then delays and final position discrepancies can be reduced leading to higher success rate of the missions.

Overall, the fundamental problem of "best" intelligence split in a multi component system is immediately hit with low cost equipment used here because for given performance level there always exists a limit beyond which uncertainty ball cannot be covered by robustness ball produced by performance level. This is making present analysis a very useful one for studying this critical limit and its determination.

\section{ACKNOWLEDGMENT}

The authors would like to thank ECE Paris School of Engineering and ONERA for having provided the environment for the development of research process. They express their gratitude to J. Marzat and S. Bertrand for assistance provided at all steps of the project. They also acknowledge Pr M. Cotsaftis for assistance in writing the manuscript. 


\section{REFERENCES}

[1] E. Montijano, D. Zhou, M. Schwager, and C. Sagues, "Distributed formation control without a global reference frame," in Proc. ACC 14, Portland, pp. 3862-3867, 2014

[2] A. S. Huang, A. Bachrach, P. Henry, M. Krainin, D. Maturana, D. Fox, and N. Roy, "Visual odometry and mapping for autonomous flight using an RGB-D camera," in Proc. Int. Symposium on Robotics Research, Aug. 2011.

[3] J. Pestana, I. Mellado-Bataller, C. Fu, J. L. Sanchez-Lopez, I. F Mondragon, and P. Campoy, "A general purpose configurable navigation controller for micro Aerial Multirotor vehicles," in Proc. 2013 Intern. Conf. on Unmanned Aircraft Systems, pp. 557-564, 2013

[4] L. Mejias and I. F. M. P. Campoy, "Omnidirectional bearing-only see-and-avoid for small aerial robots," in Proc. 5th Intern. Conf. on Automation, Robotics and Applications, pp. 23-28, 2011.

[5] Y. Rochefort, "Guidage coopératif utilisant la règle des plus proches voisins," Thesis, Supélec, 2014.

[6] Y. Rochefort, "Guidage coopératif utilisant la commande prédictive," Thesis, Supélec, 2014.

[7] B. D. Newton, "Executing RRT paths with the A R. drone quadrotor," University of North Caronlina at Chapel Hill, 2012.

A. Wendel, M. Maurer, A. Irschara, and H. Bischof, "3D vision applications for MAVs: Localization and reconstruction," in Proc. Intern. Symp. on 3D Data Processing, Visualization and Transmission (3DPVT), pp. 3-4, 2011.

[8] J. Engel, J. Sturm, and D. Cremers, "Scale-aware navigation of a low-cost quadrocopter with a monocular camera," Robotics and Autonomous Systems, pp. 1646-1656, 2014.

[9] J. Engel, "Autonomous camera-based navigation of a quadrocopter," Master Thesis, Technical Univ. Munich, 2011.

[10] G. M. Hoffmann, S. L. Waslander, and C. J. Tomlin, "Quadrotor Helicopter Trajectory Tracking Control, Proc.AIAA Guidance, Navigation and Control Conference and Exhibit, Honolulu, Hawaii, USA, August 2008 (equ du movement).

[11] A. Bachrach, R. He, and N. Roy, "Autonomous flight in unstructured and unknown indoor environments," Intern. J. Micro Air Vehicles, vol. 1 pp. 217-228, 2011.

[12] G. Klein and D. Murray, "Parallel tracking and mapping on a camera phone," in Proc. 2009 8th IEEE International Symposium on Mixed and Augmented Reality, pp. 83-86, 2009.

[13] E. Mouragnon, F. Dekeyser, P. Sayd, M. Lhuillier, and M. Dhome, "Real time localization and 3d reconstruction," in Proc. IEEE Intl. Conference on Computer Vision and Pattern Recognition, pp. 363-370, New York, 2006.

[14] F. Leberl, A. Irschara, T. Pock, P. Meixner, M. Gruber, S. Scholz, A. Wiechert, and S. Scholz, Point Clouds: Lidar versus $3 D$ Vision,
Photogrammetric Engineering and Remote Sensing, vol. 76, no. 10, pp. 1123-1134, 2010.

[15] J. Pestana, J. L. Sanchez-Lopez, I. Mellado-Bataller, F. Changhong, and P. Campoy, AR Drone Identification and Navigation Control at CVG-UPM, 2012.

[16] J. Engel, J. Sturm, and D. Cremers, "Accurate figure flying with a quadrocopter using onboard visual and inertial sensing," in Proc. IROS Workshop on Visual Control of Mobile Robots (ViCoMoR), pp. 43-48, 2012.

[17] G. Klein and D. Murray, "Parallel tracking and mapping for small AR workspaces," in Proc. Intern. Symp. on Mixed and Augmented Reality (ISMAR-07), 2007.

[18] I. Mellado-Bataller, J. Pestana, M. A. Olivares-Mendez, P. Campoy, and L. Mejias, "M"AVWORK: A framework for unified interfacing between micro aerial vehicles and visual ccontrollers," Frontiers of Intelligent Autonomous Systems Studies in Computational Intelligence, vol. 466, pp. 165-179.

[19] M. Bennewitz, W. Burgard, A. Hornung et al., "OCTOMAP: An efficient probabilistic 3D mapping framework based on octrees," Autonomous Robots. G. Sukhatme, Springer US, pp 189-206.

[20] J. H. Gillula, H. Huang, M. P. Vitus, and C. J. Tomlin, "Design of guaranteed safe maneuvers using reachable sets: Autonomous quadrotor aaerobatics in theory and practice," in Proc. IEEE Intern. Conf. on Robotics and Automation, 2010, pp. 1649-1654.

[21] Y. Rochefort, and M. P. L. G. Coopératif, "Cooperative guidance of UAVs for area exploration with final target allocation," in Proc. 19th IFAC Symp. on Automatic Control in Aerospace, Würzburg, Germany, Sept. 2-6, 2013.

[22] N. Dijkshoorn, "Simultaneous localization and mapping with the AR drone," Master Thesis, Universiteit van Amsterdam, July 2012.

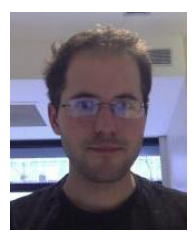

Vincent Germain started in the late 90's by conceiving websites from scratch, and then continued for years in order to acquire a deep knowledge of the web of today (coding, content writing, seo, ...). He is a graduate studies pushed his technical skills to a higher level (memory management, algorithms, neural networks,). But especially, Vincent Germain had the opportunity to work on more "research and development" projects with a very practical approach (remote sensing, exploration robots, handicap sector, ...). It was only at the end of my studies that Vincent Germain started to work in the mobile development industry: first through personal projects, then by the creation of a course for my school, and lastly by joining Appsfire. 